\title{
Computer Simulation of the Reliability of Wire Bonds and Ribbon Bonds in Power Electronics Modules
}

\author{
Kenneth Chimezie Nwanoro*, Hua Lu, Chunyan Yin, Chris Bailey, \\ Computational Mechanics and Reliability Group, University of Greenwich, London, UK \\ * Corresponding Author: K.C.Nwanoro@gre.ac.uk, +44 7592539071
}

\section{Abstract}

Aluminium wires are widely used in power electronics modules to connect power semiconductor devices and other parts of the module electrically. Recently, other interconnect techniques have been proposed such as ribbon bond to improve the reliability, performance and reduce costs of power modules. The reliability of ribbon bond technique for an IGBT power module under power cycling is compared with that of conventional wire bond in this study using electro-thermal nonlinear Finite Element Analysis. The results showed that a single ribbon of $2000 \mu \mathrm{m} \times 200 \mu \mathrm{m}$ will replace three wire bonds of $400 \mu \mathrm{m}$ in diameter to achieve a similar module temperature distribution under same power load. Using the equivalent plastic strain increment per cycle, it is seen that the ribbon bond is more reliable than the wire bonds. The impact of neglecting joule heat in the wire/ribbon bonds during power cycling simulation has also been investigated.

\section{Introduction}

Power electronics systems using semiconductors such as metal oxide semiconductors field effect transistors (MOSFET), insulated gate bipolar transistors (IGBT) and diodes are increasingly being used in many applications ranging from electrical traction applications in elevators, electric vehicles, subways and locomotives, power generation and transmission, computing, health care, aviation and aerospace, all requiring high power density and high reliability.

To meet the increasing demand for high power density power electronic systems with higher current carrying capability and switching frequency while at same time moving towards miniaturisation, low-cost and reduced weight trends as well as reducing some inherent failure mechanism of power electronic components, power electronics designers and manufacturers are devising new packaging methods and materials to meet such demands. This has led to development of efficient interconnect designs to meet demands such as low on state resistance [1] and high temperature operating materials. One of such measures is through changing the wire geometry by the introduction of Aluminium (Al) ribbon bonds for power chips interconnections - an alternative from the conventional round $\mathrm{Al}$ wire bonds. The advantages of the ribbon over round wire bonds are mainly the larger surface area in relation to the cross sectional area providing lower parasitic and lower impedance at high frequency. This geometric feature gives the ribbon a lower electrical resistivity than a wire of the same length and therefore a higher current handling capability as can be seen in (1) and (2). This allows less use of multiple bond wires to meet a current requirement or fulfill a necessary resistance.

$$
\begin{gathered}
R_{w}=\frac{\rho_{o} L}{A_{w}}=\frac{\rho L}{\pi r^{2}} \\
R_{r}=\frac{\rho_{o} L}{A_{r}}=\frac{\rho_{o} L}{w t}
\end{gathered}
$$

where $R_{w}$ and $R_{r}$ are wire and ribbon resistance respectively, $\rho_{o}$ is the material resistivity, $\mathrm{L}$ is the length or span of the wire/ribbon, $\mathrm{t}$ is the ribbon thickness, $\mathrm{r}$ is the wire radius $A_{r}$ and $A_{w}$ are the cross-sectional area of the wire and ribbon respectively.

Furthermore, the issue of cratering defects during wire bonding is less with $\mathrm{Al}$ ribbon than with round wire especially during initial stage of bonding process because the ribbon's larger surface area allows better distribution of bond force and ultrasonic energy [2]. Another benefit of the $\mathrm{Al}$ ribbon over wire bonding is that the reduced number of bonds means less defects during bonding process and therefore higher quality and throughput. The larger surface area of the ribbon also means that it has a better heat conduction capability and can remove joule heating generated around the chip - pad interface faster.

Due to the rectangular shape of the ribbon, a better continuous contact bonding area can easily be implemented on a bond pad die metallization thereby eliminating the non-continuous contact that is often the case when multiple wires terminate on the same bond pad which results in high current entering the semiconductor which may lead to local differences in heat dissipation as well as the electric field strength [3]. Furthermore, compared to the same surface area, less bonding force and ultrasonic power is required to deform and bond the ribbon because of its lower cross-sectional area/thickness compared to round wire.

Miniaturization of the power electronic systems is made easier by using ribbon bonding whilst increasing the power density and the frequency of power electronics applications, this is due to the fact that increasing diameter of wires for high power capability creates larger loops and a bigger 
casing. However, due to the planar nature of the ribbon, reduced thickness and lower loop height are possible for high power density application.

Ribbon bonds are used extensively for high frequency applications such as optical and optoelectronic devices and in the past recent years, have been developed for power electronic applications such as in compact DC-DC MOSFET converters like $D^{2}$ PAK and quad-flat-no-lead (QFN) packages [4] but to the author's knowledge has not been used for a commercial IGBT power module.

\subsection{Wire bond failure modes}

Figure 1 shows the two major failure modes: bond wire lift-off at bond foot and heel crack [5]. The bond wire lift-off happens more frequently.

The wire bond failure is mostly due to thermo-mechanical fatigue from temperature swings that are resulted from power dissipation in the power semiconductors during switching and conduction, and from heat generation in the wires due to ohmic heating. During the operation of a power electronic system, high electric current flow from one region of a power module device to another, especially from the emitter terminal to the diode anode connection. The currents cause temperature increase that result in stress concentration at the interface between the bond wire and the bond pad which may lead to horizontal cracks and failures at the bond interface [6]. When the wires are heated repeatedly, the flexure of the wire may lead to vertical crack and failure at the heel [7]. The thermo-mechanical stresses are intensified due to the CTE mismatch between the $\mathrm{Al}$ bond wire and the silicon chip that it is bonded.

The effect of wire bond failure results in changes in either contact resistance value or internal distribution of current leading to increased temperature above the safe operating area $(\mathrm{SOA})$.

The thermo-mechanical behaviour of gold ribbon used in optoelectronics application has been investigated by Tan et al [8] using pull and shear tests under different temperature ranges and aging time. This study showed that ribbon bonds have higher pull and shear strength than round wire bonds. Celnikier et al [5] performed reliability tests for $D^{2}$ PAK package using passive thermal cycling for ribbon bond and compared with wire bonds. In [9], the mechanical properties of Al-ribbon bonds such as tensile strength at different temperatures were investigated and using pressure cooker tests for high temperature storage and aging tests for reliability were performed. The high temperature storage tests showed low intermetallic compound (IMC) of $\mathrm{Al}_{2} \mathrm{Cu}$ formation as result of low diffusion and concluded that the risk of ribbon bond failure on direct copper bonding (DBC) caused by diffusion is very low, while the pull tests after thermal storage tests decreases with storage time caused by recrystallization softening of $\mathrm{Al}$ and copper $\mathrm{Cu}$.

The limitation of these studies is that the reliability of the ribbon bonds based on heat generation from Joule heating and cyclic power loss in the power device especially for IGBT power modules has not been investigated and compared with wire bonds, therefore, its real failure modes and mechanism has not been characterized. Hence, the need to model its thermo-mechanical behaviour and compare with the conventional round wire bonds for IGBT modules under power cycling as well investigate the impact of neglecting the wire joule heating during power cycling simulation (as this is often the case in the literature) on the computed number of cycles to failure.

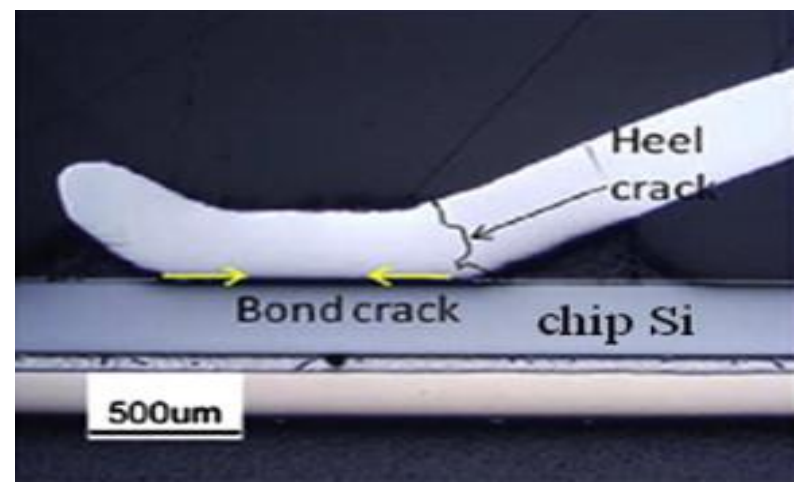

Figure 1: Failure modes in wire bonds

\section{Methodology}

Two IGBT power modules models are shown in the Figures 2 and 3 with wire bond and ribbon bonds respectively. They are analysed in this study using the Finite Element Method (FEM). The IGBT power module dimensions, layout and characteristics are similar to that of Powerex IGBT halfbridge module CM150DU-24NFH- a commercial power IGBT module [10].

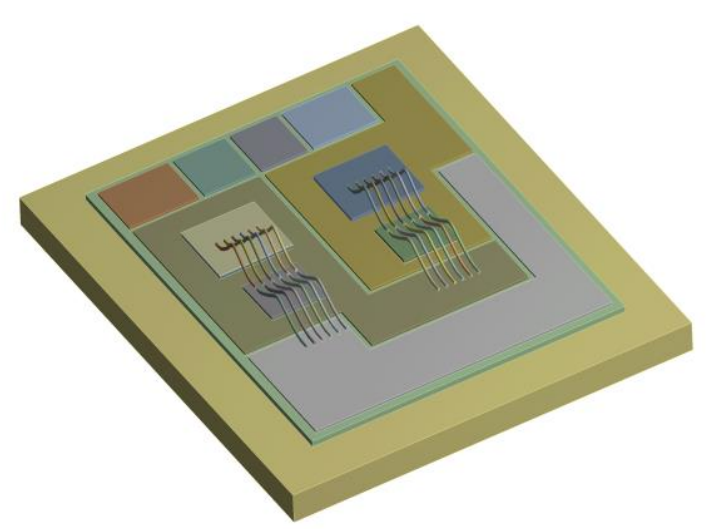

Figure 2: IGBT module with wire bonds 


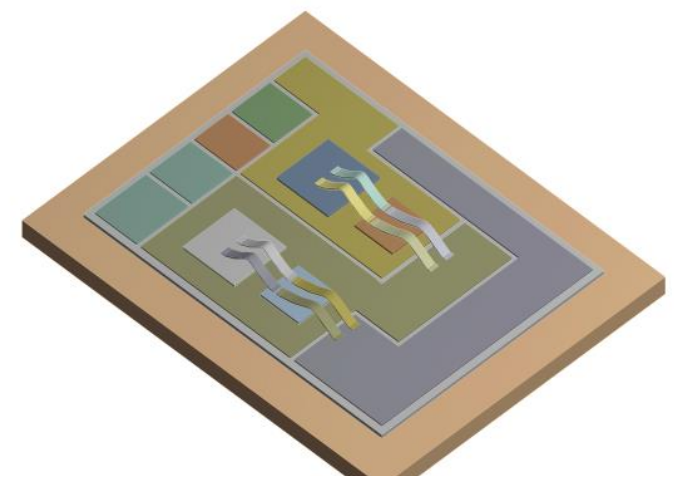

Figure 3: IGBT module with ribbon bonds

The IGBT power module is made of several layers of different materials as shown in the Figure 4.

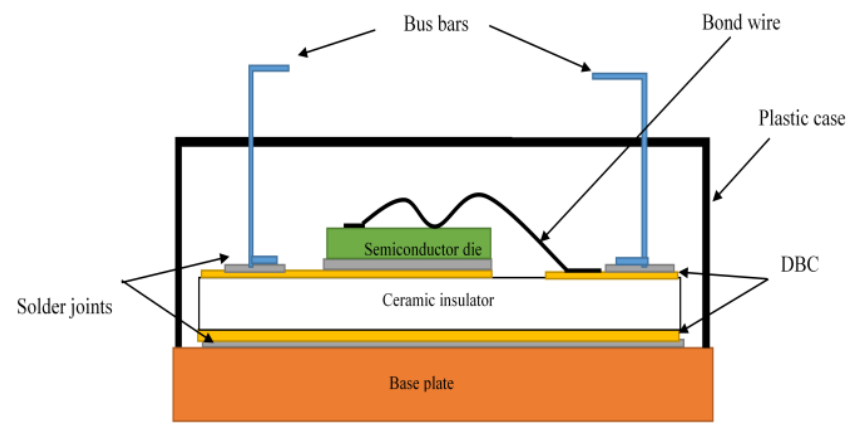

Figure 4: Schematic of IGBT power module

\subsection{Finite element analysis (FEA) simulation}

The power cycling simulation was performed using FEA and a commercial multi-physics FEM software ANSYS has been used. Power cycling is realized by switching heat generation in the module on and off using the second-level [11] method since the study is focused on the wire/ribbon bonding with a constant turn on (heating) and turn off (cooling) times of 4 seconds and 11 seconds respectively giving a 15 seconds cycle period.

In the simulation, a power loss of $207 \mathrm{~W}$ and $175 \mathrm{~W}$ are applied to the IGBT power chips and diodes respectively which corresponds to 100 amperes of current density in the wires or ribbons.

At the bottom of the baseplate, the convective heat transfer coefficient value is $2000 \mathrm{~W} / \mathrm{m}^{2} \mathrm{C}^{\mathrm{o}}$ during cooling and $5 \mathrm{~W} / \mathrm{m}^{2} \mathrm{C}^{\mathrm{o}}$ during heating. Two loading conditions are applied. In the first one heat generation is applied in the chip only and in the second one it is also applied to the wires/ribbons giving 4 cases. Case 1 and 2 is the ribbon bond and wire bond without joule heating while case 3 and 4 is the ribbon bond and wire bond with joule heating respectively.

All the materials are modelled as linear elastic with exceptions of solder and the aluminium wire/ribbon. The solder material is $96.5 \mathrm{Sn} 3.5 \mathrm{Ag}$ and it has been modelled as a viscoplastic material using the Anand's model. The aluminium wire/ribbon bonds have been modelled as elasticplastic material with isotropic hardening. The material properties used in this study is presented in Table 1 and obtained from [12] and the Anand's parameters [12] is given in Table 2.

Considering the size of the models, a sub-modelling technique was adopted whereby, the full model was modelled with coarse mesh and then a section of interest (wire and ribbon bonds) was taken and refined with a very fine mesh.

\subsection{Thermo-Mechanical analysis}

In FEM, thermo-mechanical analysis can be performed as direct (fully) coupled phenomenon by solving simultaneously the strain-heat transfer (3) and temperature/displacement (4) equation using a coupled element while considering material nonlinearities.

$$
\begin{array}{r}
\nabla .(k \nabla T)-\rho C \frac{d T}{d t}-(3 \lambda+2 \mu) \dot{\varepsilon}_{i i} \alpha T_{0}=-\dot{q} \\
\mu u_{i, k k}+(\lambda+\mu) u_{k, k i}-(3 \lambda+2 \mu) \alpha \Delta T=0
\end{array}
$$

where $k$ is the material thermal conductivity, $\rho$ is the density, $C$ is the specific heat capacity, $\dot{q}$ is the heat generation rate, $u$ is the displacement, $\varepsilon$ is the strain, $\lambda$ and $\mu$ are the Lame's constants and $\Delta T$ is the temperature change from a zero strain reference temperature.

In this study, sequentially coupled thermal-stress analysis has been used. The temperature distributions in the power module as a result of the heat dissipation and joule heating is computed for a transient thermal analysis using (5) while (6) is used to compute the joule heat in the wire/ribbon.

$$
\begin{gathered}
\nabla .(k \nabla T)+\dot{q}=\rho C(d T) / d t \\
\mathrm{Q}=I^{2} \mathrm{R}=I^{\wedge} 2 \rho_{o} L / A
\end{gathered}
$$

where: $\mathrm{Q}$ is the wire/ribbon joule heat.

Then, the temperature distribution is used as a thermal load to compute the thermal force vector (7) which is used to calculate the transient stress and strain distribution in the power module.

$$
F_{t h}=E \alpha \Delta T
$$

where $\mathrm{E}$ is the young's module and $\alpha$ is the CTE. When discretised over the domain in FEM formulation, (7) becomes

$$
F_{t h}=\int B^{T} D\{\alpha \Delta T\} d v
$$

where $B^{T}$ is the transpose of the derivatives of element shape functions, D is linear isotropic fourth order Hooke's tensor. 
Table 1: Material properties.

\begin{tabular}{|c|c|c|c|c|c|}
\hline Material properties & Silicon & $\mathrm{Al}$ & $\mathrm{Cu}$ & $\begin{array}{c}\text { Aluminium } \\
\text { Nitride }\end{array}$ & Solder \\
\hline Young's modulus (GPa) & 113 & 70 & 103.42 & 310 & $54.05-0.19 \mathrm{~T}$ \\
\hline Poisson's ratio & 0.29 & 0.33 & 0.3 & 0.24 & 0.4 \\
\hline CTE $(1 / \mathrm{K})$ & 2.6 & 23.1 & 17 & 5.6 & $21.85+0.02039 \mathrm{~T}$ \\
\hline Electrical resistivity $(\Omega . m)$ & 0.0001 & $2.65 \times 10^{-8}$ & $1.7 \times 10^{-8}$ & $1 \times 10^{14}$ & $1.3 \times 10^{-7}$ \\
\hline Density $\left(\mathrm{kg} / \mathrm{m}^{3}\right)$ & 2330 & 2700 & 8930 & 3300 & 8400 \\
\hline Specific heat $(\mathrm{J} / \mathrm{kg} K)$ & 729 & 950 & 400 & 740 & 150 \\
\hline
\end{tabular}

Table 2: Material parameters for the Anand viscoplastic model.

\begin{tabular}{|c|c|c|c|c|c|c|c|c|c|}
\hline Parameters & $S_{o}(\mathrm{MPa})$ & $\mathrm{Q} / \mathrm{R}(\mathrm{K})$ & $\mathrm{A}\left(s^{-1}\right)$ & $\xi$ & $m_{0}$ & $h_{0}(\mathrm{MPa})$ & $\hat{s}(\mathrm{MPa})$ & $\mathrm{n}$ & $\mathrm{a}$ \\
\hline Solder & 39.09 & 11262 & $2.23 \times 10^{4}$ & 6 & 0.182 & 3321.15 & 73.81 & 0.0018 & 1.82 \\
\hline
\end{tabular}

\subsection{Life Prediction model}

The life prediction model used for predicting the number of cycles to failure $N_{f}$ is given in (9) and is based on the equivalent plastic strain increment per cycle $\Delta \bar{\varepsilon}^{p}$ [9].

$$
N_{f}=B_{1} \Delta \bar{\varepsilon}^{p B_{2}}
$$

where $B_{1}$ and $B_{2}$ are constants given in Table 3 .

Table 3: Constants for life prediction model [9].

\begin{tabular}{|c|c|}
\hline$B_{1}$ & $B_{2}$ \\
\hline 113.189 & -1.223 \\
\hline
\end{tabular}

\section{Results \& Discussion}

The temperature history for power devices (juncture) for case 1 and 2 under power cycling is shown in Figure 5 while a typical temperature distribution in the power module at the end of the heating time in shown in Figure 6. The Figure 3 shows that the temperature history is similar for the two power module designs. The average temperature history in the power chips for the four cases is shown in Figure 8 while Figure 7 shows the average temperature histories for the wire and ribbon bonds. It is seen, that average chip temperature is similar in all cases with about 1 to $2{ }^{\circ} \mathrm{C}$ increase in temperature for case 3 and 4 .

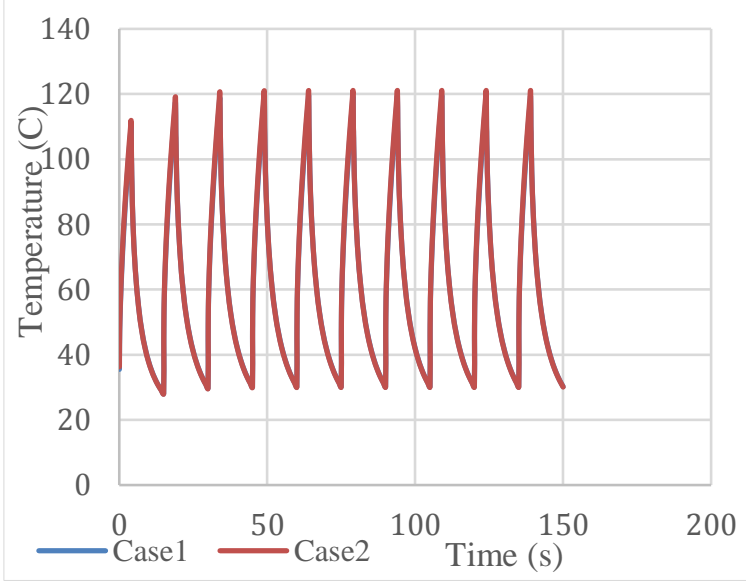

Figure 5: IGBT juncture temperature history for casel and 2

However, the introduction of the joule heat obviously increases the average ribbon and wire bond temperatures as is seen in Figure 7. 


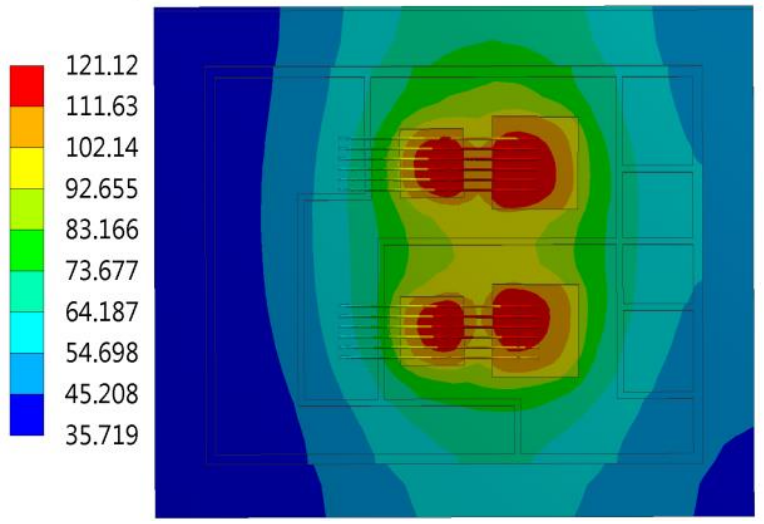

Figure 6: IGBT power module temperature distribution for case 1 .

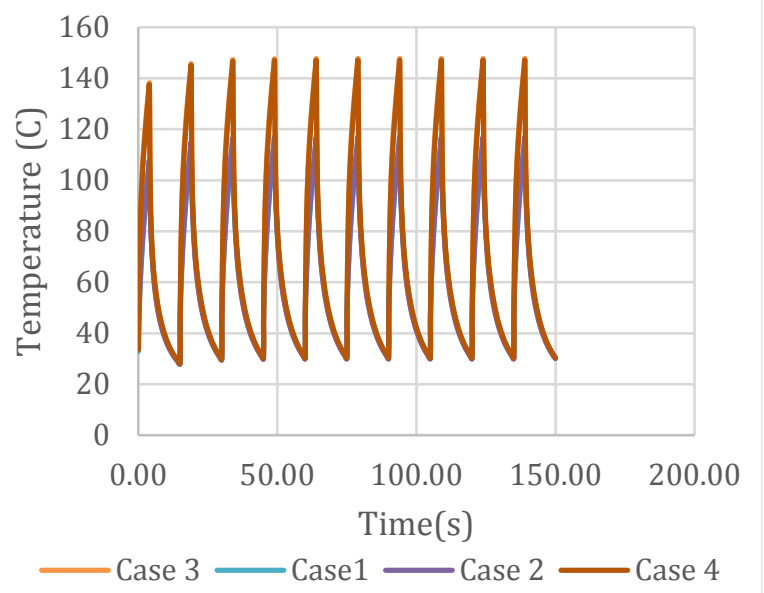

Figure 7: Average wire and ribbon temperatures

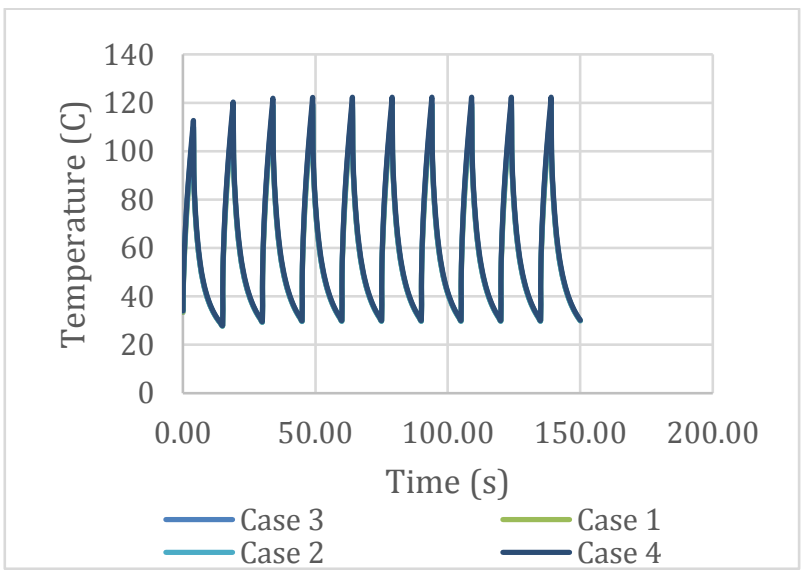

Figure 8: Average power chip temperature for all cases

The Figures 9 and 10 show the equivalent plastic strain distribution in the ribbon and wire bond respectively at the end of the power cycling.

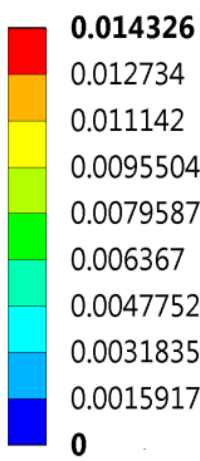

Figure 9: Equivalent plastic strain distribution in the ribbon

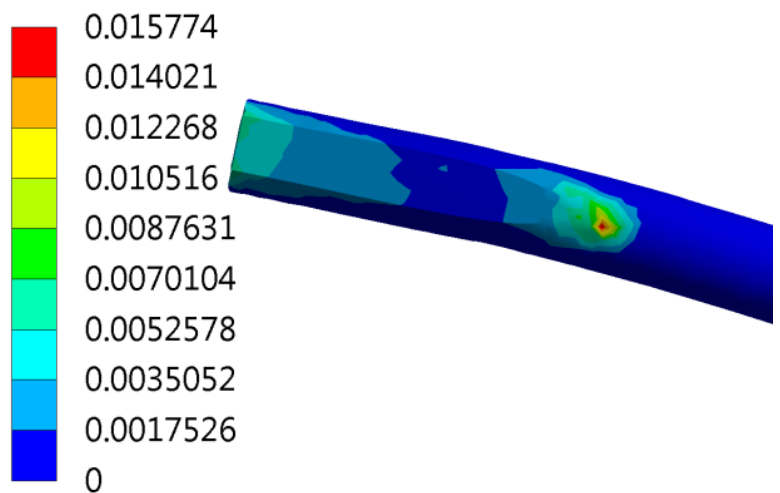

Figure 10: Equivalent plastic strain distribution in the wire

The Figure 9 showed that equivalent plastic strain distribution is concentric in the ribbon bond and maximum at the corners where crack initiation is expected to occur and propagate inwards leading to possibly bond lift being the dominant failure as a result of CTE mismatch. The equivalent plastic strain distribution in the wire bond is maximum at the heel and also distributed at the bond interface showing a heel crack dominant failure mode due to wire flexure as well as a bond wire lift off failure mode. The Figure 11 shows that equivalent plastic strain is always higher in the wire bond than in the ribbon for both loading cases and then using the life prediction model and equivalent plastic strain increment per cycle, the ribbon bond has more number of cycles to fail than the wire bond as shown in the Table 4 .

Table 4: Computed number of cycles to failure for each case.

\begin{tabular}{|l|c|c|c|c|}
\hline & Case 1 & Cas2 & Case3 & Cas4 \\
\hline$N_{f}$ & 7922 & 7339 & 6381 & 5529 \\
\hline
\end{tabular}




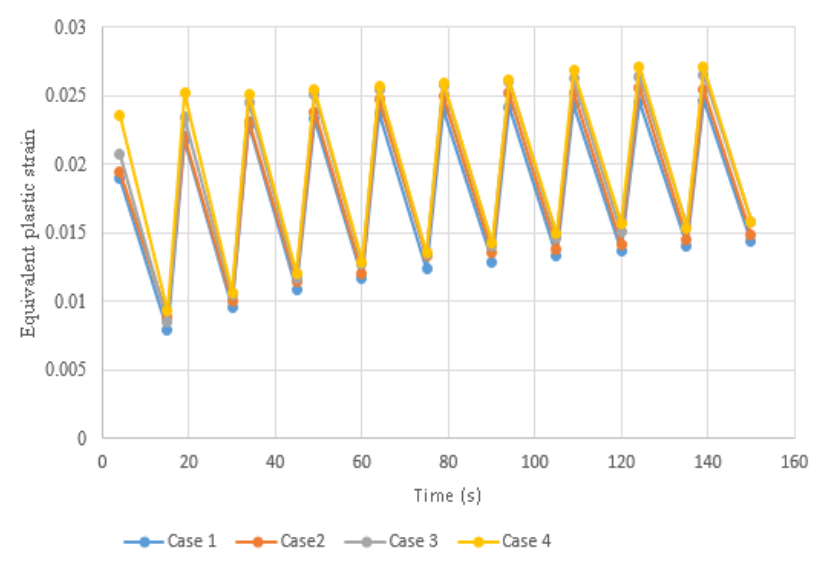

Figure 11: Equivalent plastic strain history in the wire and ribbon bonds for all cases

\section{Acknowledgements}

Kenneth Chimezie Nwanoro is funded by the University of Greenwich's Vice Chancellor's PhD Scholarship Scheme.

\section{Conclusion}

The results have shown that if a single ribbon of $2000 \mu \mathrm{m}$ by $200 \mu \mathrm{m}$ is used to replace three $400 \mu \mathrm{m}$ diameter wires, the temperature in the ribbon and wires will be almost identical. The stress and strain distribution in the ribbon bond shows that bond lifting is the dominant failure mechanism whereas heel cracking is also a significant failure mechanism in conventional wire bonds because of wire flexing. This shows that the ribbon bonding could not only be more economical for large current capability but also more reliable than wire bonding. This research has also found that in the simulation, it is important to consider the joule heating in the wire in addition to the heat dissipation in the chips during power cycling simulation.

\section{Literature}

[1] B. Ong, M. Helmy, and S. Chuah, "Heavy Al ribbon: An alternative solution for hybrid power packaging," IMAPS, Long Beach, CA, pp. 1-11, Nov., 2004.

[2] W.Q. Ivy, P. Reid, R.E. Werner, and D. Doerr, "Automatic wedge bonding with ribbon wire for high frequency applications," $27^{\text {th }}$ IEEE/SEMI International electronics manufacturing technology symposium, IEMT, pp. 97-104, July, 2002.

[3] R. Gilardoni, "Ribbon bonding for high frequency applications: Advanatges of ribbon and impact on the microwave market," IMAPS/SEMI Advanced technology workshop on wire bonding, San Francisco, pp. 1-5, July 14, 2008,

[4] S. Jacques, R. Leroy and M. Lethiecq, "Impact of aluminium wire and ribbon bonding technologies on $D^{2} P A K$ package reliability during thermal cycling application," Microelectronics reliability, Elsevier, vol. 55 , pp. 1821-1825.
[5] Y. Celnikier, L. Dupont, E. Herve, G. Coquery and L. Benabou, "Optimization of wire connections design for power electronics, Journal of Microelectronics reliability, vol. 51, no. 9-11, pp. 1892-1897, Elsevier, 2011.

[6] S. Ramminger, G. Mitic, P. Türkes and G. Wachutka, "Thermo-mechanical simulation of wire bonding joints in power modules," International conference on modelling and simulation of microsystems, San Juan, Puerto, pp. 483-486, April, 1999.

[7] M. Ciappa, "Selected failure mechanism of modern power modules," Microelectronics reliability, vol. 42, no. 4-5, pp. 653-667, May, 2002.

[8] C.W. Tan, Y.C. Chan, H.D. Liu and B.N.M. Leung, "A study on the reliability and thermos-mechanical properties of gold ribbon wire bonding," IEEE Electronics components and technology conference, vol. 1, pp. 377-382, 2004.

[9] E. Milke and T. Mueller, "High temperature behavior and reliability of $\mathrm{Al}$ ribbon for automotive applications," $2^{\text {nd }}$ IEEE Electronics system-integration technology conference, Greenwich, UK, pp. 417-422, 2008.

[10] Powerex Corporation. (2013). Datasheet of NFH-series IGBT module (150amperes/1200volts) [Online]. Available: ttp://www.pwrx.com/pwrx/docs/cm150du24nfh.pdf

[11] T-Y. Hung, L-L.Liao, C.C. Wang, W.H. Chi and K-N. Chiang, "Life prediction of high-cycle fatigue in aluminum bonding wires under power cycling test," IEEE Transactions on Device and Materials Reliability, vol.14, no. 1, pp. 484-492, March, 2014.

[12] P. Rajaguru, H. Lu and C. Bailey, "Application of nonlinear fatigue damage models in power electronic module wirebond structure under various amplitude loadings, Journal of advances in manufacturing, vol. 2, no. 3, pp. 239-250, 2014.

[13] A. Wright, A. Schletz and A. Pichler, "Thermomechanical simulation of plastic deformation during temperature cycling of bond wires for power electronic module," $15^{\text {th }}$ International conference on thermal, mechanical and multi-physics simulation and experiments in microelectronics and microsystems, EuroSimE, pp. 1-5, 7-9 April, 2014, IEE.

[14] G.Z. Wang, Z.N. Cheng, K. Becker and J. Wilde, "Applying Anand model to represent the viscoplastic deformation behavior of solder alloys," ASME Journal of Electronic packaging, vol. 123, pp.247-253, 2001. 